\title{
Simultaneous Data Collection of SMall Maritime Targets using MULTISTATIC AND FORWARD SCATTER RADAR
}

\author{
Matthew Ritchie ${ }^{1}$, Francesco Fioranelli ${ }^{3}$, Karl Woodbridge ${ }^{1}$, Hugh Griffiths ${ }^{1}$, Liam Daniel ${ }^{2}$, Alessandro \\ De Luca ${ }^{2}$, Stanislav Hristov ${ }^{2}$, Marina Gashinova ${ }^{2}$, Mikhail Cherniakov ${ }^{2}$ \\ ${ }^{1}$ Department of Electronic \& Electrical Engineering, University College London, London, UK \\ ${ }^{2}$ School of Electrical, Electronic and Systems Engineering, University of Birmingham, UK \\ ${ }^{3}$ School of Engineering, University of Glasgow, UK
}

\begin{abstract}
Radar detection of small maritime targets is of great interest in the context of coastal and port security for prevention of activities such as smuggling and piracy. Multistatic radar and forward scatter radar offer detection advantages compared with conventional monostatic systems, such as advantageous multiperspective target view for the former and target radar cross section enhancement for the latter. In this paper, experimental results are presented to characterize simultaneous measurements investigating the detection of a small inflatable boat by a multistatic and a forward scatter radar. These results are believed to be the first example of simultaneous experimental comparison of such systems.
\end{abstract}

\section{Introduction}

There is a growing interest in the detection and classification of low observable maritime targets in the context of coastal areas and harbour security, to aid in prevention of illicit activities, such as smuggling, trafficking, piracy, and terrorism. Small boats and Rigid Inflatable Boats (RIBs) made of wood, fibreglass, and rubber indeed pose an asymmetric threat as conventional radar systems may struggle to detect such small and fast moving targets against the sea clutter background [1-2]. When deploying radar sensors to counter such small difficult to detect targets various innovative technologies have been proposed including Forward Scatter Radar (FSR) and multistatic radar. In order to put forward these potential solutions quantified results of both the clutter characteristics and target detection capabilities must be analysed.

Very little information has been openly published on the detection of low observable maritime targets and analysis of their signatures, particularly in bistatic or multistatic configurations [3]. Multistatic radar systems provide potential advantages over conventional monostatic radar, such as enhanced target signatures due to multi-perspective views, the possibility of having a mobile and quickly reconfigurable network of radar nodes and potential advantageous clutter properties in terms of amplitude statistics, Doppler spread and reflectivity in given geometries [4]. FSR is also suggested as an efficient tool for maritime security [5]. It can be considered as a particular type of bistatic radar operating at very large 
bistatic angles, i.e. relatively narrow angular regions around the radar baseline. Here the target forward scatter cross section (FSCS) increases significantly compared with the monostatic and bistatic RCS and is resilient to stealth targets shaping and coatings [6]. Furthermore in FSR coherent times are long [7] and sea clutter has been shown to be well characterised by the Rayleigh distribution, unlike mono or bistatic low grazing angle clutter [8], and has a spectrum that appears invariant to radar and sea state parameters.

In this paper we present a summary of experimental results of simultaneous data collection for investigating detection of the same target (a small inflatable boat) with a multistatic radar system and a forward scatter radar system. These results are believed to be the first attempt at simultaneous experimental comparison of these different radar architectures, and represent a collaboration of research groups at University College London and the University of Birmingham. A qualitative description of the target signatures from both systems is presented in this paper as an initial stage of analysis, with the aim of investigating needs and improvements for future work and facilitating progression onto more quantitative investigation. Further to the original publication this expanded version analyses and quantifies the Signal to Clutter (SCR) values, from both radar systems, for 6 scenarios comparing when a sphere was or wasn't towed behind the small boat target.

This paper is organized as follows. Section II describes the two radar systems and the experimental setup. Section III presents the discussion of the data recorded with the multistatic and the forward scatter radar for a few examples of the joint measurements made. Section IV finally concludes the paper.

\section{Radar Systems and Experimental Measurements}

\subsection{Radar Systems}

The multistatic radar system used in this series of experiments is the three-node coherent pulse radar NetRAD, developed at UCL over the past years and applied in different radar research topics, such as sea clutter characterization and human micro-Doppler analysis [9-10]. The system uses an operational frequency of $2.4 \mathrm{GHz}$ (S-band), $+23 \mathrm{dBm}$ transmitted power, $0.6 \mu$ s pulse length, $45 \mathrm{MHz}$ bandwidth, and $1 \mathrm{kHz}$ PRF. The antennas have a gain of $24 \mathrm{dBi}$ and $10^{\circ}$ beamwidth in azimuth and elevation.

The FSR system is a multi-frequency continuous wave (CW) radar developed at the University of Birmingham and is used as part of an ongoing investigation into both target and clutter signatures in the maritime environment [5-8]. It consists of a single multi-frequency transmit node and a corresponding receive node. Experimental results presented here are recorded with transmit frequencies of $7.5 \mathrm{GHz}$ and $24 \mathrm{GHz}$. The transmitter output power at both frequencies is $+26 \mathrm{dBm}$ and both channels utilise $20 \mathrm{~dB}$ horn antennas with $20^{\circ}$ beamwidth in azimuth and elevation.

\subsection{Experimental Setup}


The experiments took place at Langstone Harbour, near Portsmouth, UK, at the beginning of February 2015. The experimental geometry used is shown in Fig. 1. The three NetRAD nodes were deployed along a linear baseline with the monostatic transceiver node at one end, and the two multistatic receiver-only nodes separated by approximately $50 \mathrm{~m}$ each. The two nodes of the FS system were deployed one on each side of the Langstone harbour channel, the receiver node co-located with the middle NetRAD node. Radar antenna heights above the sea surface varied between 1 and $2.5 \mathrm{~m}$ during the trial duration due to tidal effects. The width of the channel is approximately $380 \mathrm{~m}$. This figure is a simplified sketch, as in reality the coastline is not perfectly straight and the channel has a variable width across its length. The cooperative target used for the experiments was a GPS-instrumented inflatable boat operated by the University of Birmingham team and shown in Fig. 2. The target crossed the FSR baseline several times while moving upstream and downstream in the channel, at several velocities, and multiple simultaneous recording of these movements were captured by the two radar systems. In most recordings the target crossed the baseline perpendicularly, but also crossings at certain angles were recorded. In some, an inflatable ball of $0.9 \mathrm{~m}$ diameter was towed behind the boat to generate a 'convoy' of varied RCS/FSCS targets Figure 3. During the experiments, data from two targets of opportunity were also recorded, namely a large ship and a RIB of slightly larger dimension and faster speed than the inflatable. The antenna beams of the multistatic radar were pointed to a common patch of sea in the middle of the channel on the FS baseline, where the target was expected to cross. Fig. 4 shows photos of the experimental location and part of the two systems, namely the NetRAD monostatic node with the transmitter and receiver antenna in Fig. 4a and the FS transmitting node in Fig. 4b.

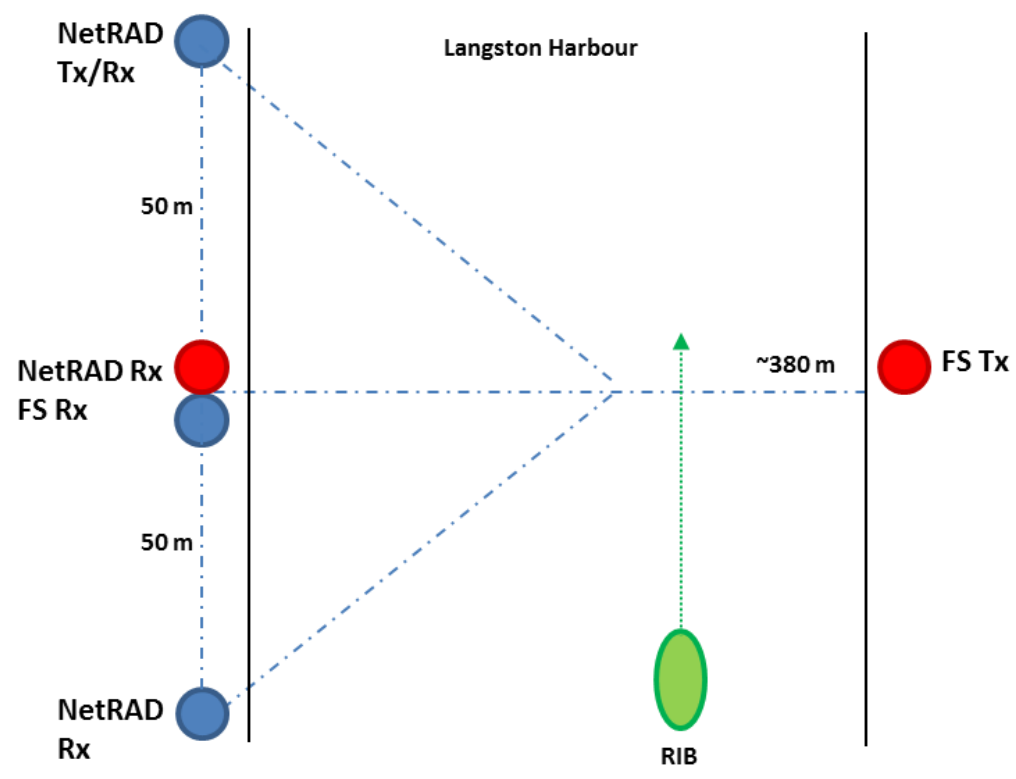

Figure 1 Diagram of experimental setup 


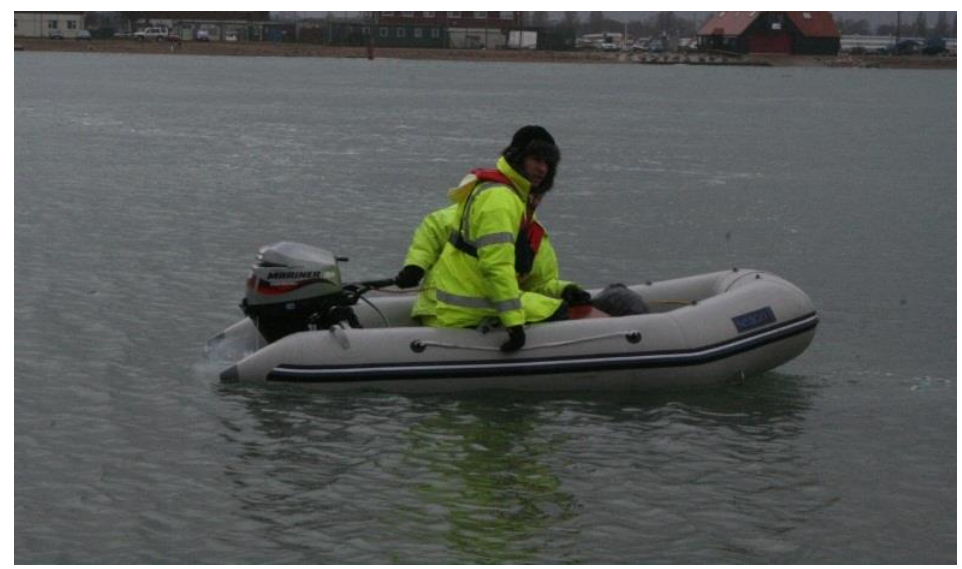

Figure 2 Small Inflatable boat used as co-operative target

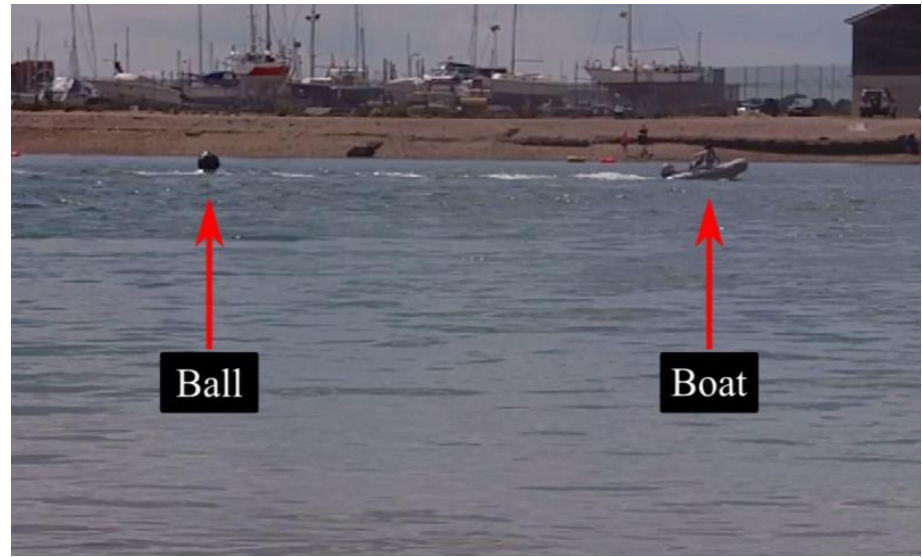

Figure 3 Towed inflatable ball, $0.9 \mathrm{~m}$ diameter

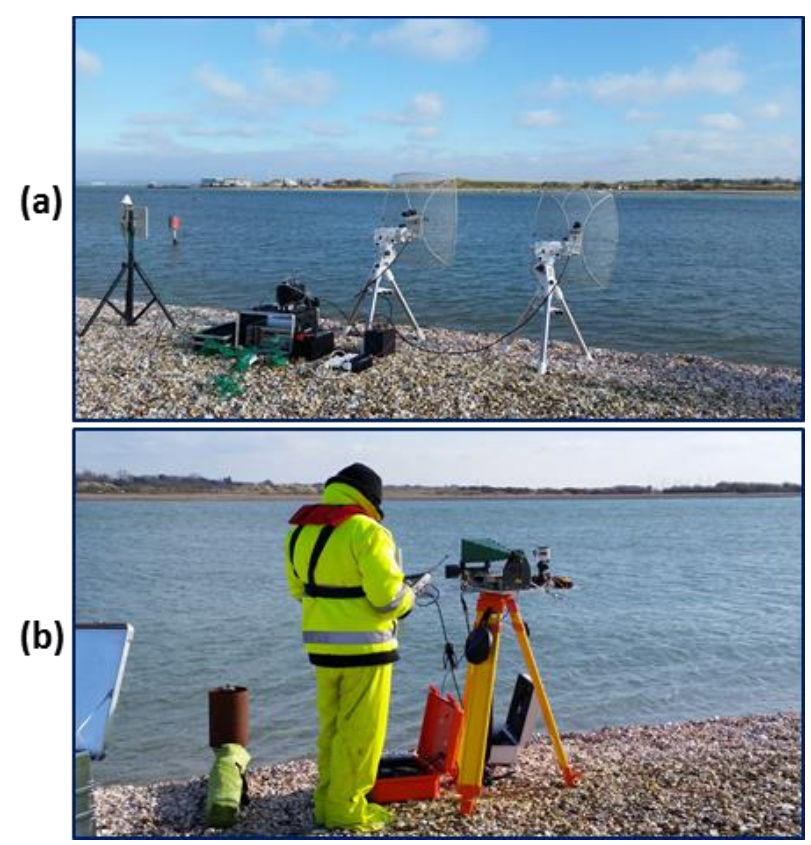

Figure 4 Experimental locations with (a) NetRAD and (b) FSR transmitter node 


\section{Data Collection and Initial Analysis}

In this section we present a number of example experiments recorded during the joint measurements and the results of the analysis. Firstly, a description of the format of the data and initial analysis for each radar system is described, followed by the descriptions of the scenarios for the then presented data. Each data set presented also has a qualitative description accompanying it.

\subsection{Data format and analysis}

Each dataset recorded using the NetRAD system consisted of 30000 pulses, corresponding to 30 seconds of data at $1 \mathrm{kHz}$ PRF. These data are presented in the form of Range-Time-Intensity (RTI) plots for each node. The target is not present in these plots for the whole duration of the recording, but only while the small inflatable boat is moving within the main beam of the transmitter and receiver antennas. The Short

Time Fourier Transform (STFT) was also applied to these data to analyse the micro-Doppler signature of the target. The STFTs were calculated with a $0.4 \mathrm{~s}$ Hamming window and $95 \%$ overlap, across the range bins, which contain returns from the target.

The output from the FSR radar is composed of the time domain Doppler variation (amplitude) of both the sea surface and targets in view. Due to the large bistatic angles involved, the Doppler frequencies are very low $(<100 \mathrm{~Hz}$ for the considered target velocities). This direct extraction of the very low Doppler frequency signatures means that the FSR receiver output is sampled at a relatively low rate of $200 \mathrm{~Hz}$, allowing for long data sets to be collected if required. The results displayed in the following sub-sections are from the 7.5 and $24 \mathrm{GHz}$ radar channels, and their accompanying spectrograms (STFTs) which provide a more visually interpretable representation of the characteristic double-sided chirp signal as the target approaches and crosses the baseline (crossing at $0 \mathrm{~Hz}$ Doppler). It should be noted that due to the current system design, all Doppler frequencies are measured as positive. Minimal processing has been applied to the signals, consisting of subtraction of the mean level for removal of the signal DC level which is present in all signatures due to influence of the direct path signal between facing transmitter and receiver.

The next subsections outline a selection of the scenarios measured and the corresponding data collected from both radar systems.

\subsection{Experiment 1 - Perpendicular mid-point crossing of FSR baseline}

The first joint measurement was that of the small inflatable moving upstream perpendicularly crossing the middle of the FSR baseline. The target trajectory is shown in Fig. 5, indicating the FSR baseline (red line) formed between the transmitter and receiver. The speed for the crossing as measured by GPS was on 
average $4 \mathrm{~ms}^{-1}$ when approaching the baseline, it was noticed in the GPS data however that the speed then increased to an average $7 \mathrm{~ms}^{-1}$ post crossing.

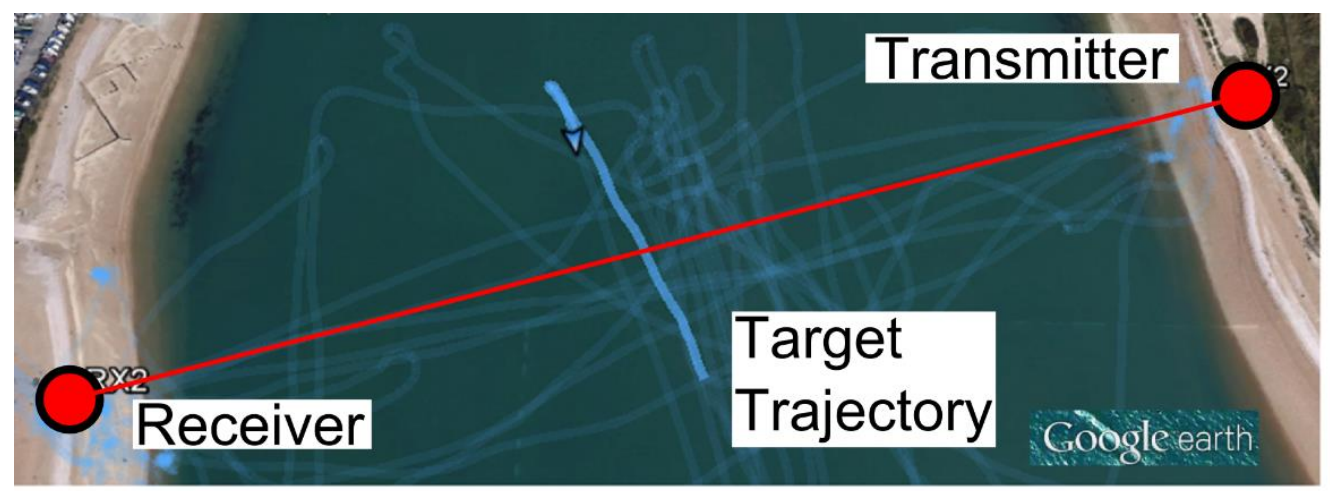

Figure 5 GPS track showing target trajectory for first described experimental data.

Fig. 6 shows the RTI plots for the multistatic radar as recorded at the three nodes. Fig. 7 shows the corresponding micro-Doppler signatures of the RTI plots and Fig. 8 shows the Doppler signatures and corresponding spectrograms for both FSR channels. In Fig. 6a the small inflatable boat is clearly visible (monostatic plot) at the beginning of the recording, it then gets more visible in all three plots at approximately $350 \mathrm{~m} \mathrm{2-way} \mathrm{range} \mathrm{as} \mathrm{it} \mathrm{moves} \mathrm{across} \mathrm{the} \mathrm{patch} \mathrm{covered} \mathrm{by} \mathrm{the} \mathrm{antenna} \mathrm{beams} \mathrm{from} \mathrm{all}$ nodes, and then it is no longer detected as it moves out of this patch. The reflection from the coastline at the other side of the harbour channel is also visible as a bright line at approximately $760 \mathrm{~m}$ 2-way range, corresponding to the physical channel width of approximately $380 \mathrm{~m}$. The direct signal from the monostatic transmitter is also visible in Fig. $6 \mathrm{~b}$ and $6 \mathrm{c}$, with data from the multistatic receiver at node 1 and node 2 , respectively. The signature of the boat is visible in the monostatic data from the beginning of the recording up until around $19 \mathrm{~s}$ (Fig. 7a), whereas in the multistatic data (Fig. 7b and 7c) it is weaker at the beginning, brighter between around $5 \mathrm{~s}$ and $17 \mathrm{~s}$, and then no longer visible after around $21 \mathrm{~s}$. The main Doppler shift is higher for the monostatic data, at approximately $17.5 \mathrm{~Hz}$ corresponding to approximately $1.1 \mathrm{~m} / \mathrm{s}$ at the $2.4 \mathrm{GHz}$ carrier, and tends to get lower for the bistatic data at node 1 (Fig. $7 \mathrm{~b}$ ), and even more at node 2 (Fig. 67c), where it gets close to zero and negative between around $14 \mathrm{~s}$ and $18 \mathrm{~s}$. Between $18 \mathrm{~s}$ and $21 \mathrm{~s}$, the main Doppler shift appears to increase in all the plots, as if the boat was moving closer to the nodes. This may be related to the imperfect perpendicular trajectory of the RIB because of the strong tide in the channel or could be caused by the boat turning around at the end of the trajectory as shown in the GPS track. 
(a)

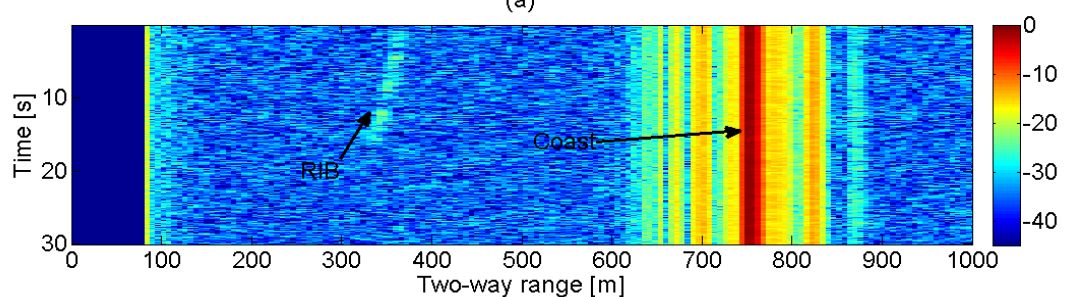

(b)

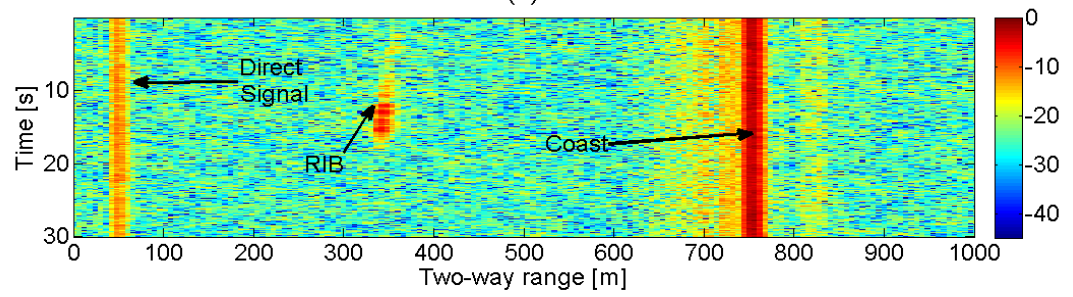

(c)

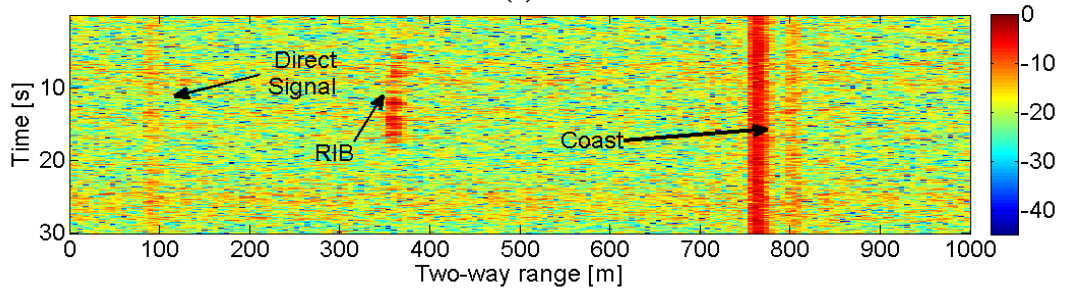

Figure 6 Range-Time plots of the first joint experiment as recorded at NetRAD node 3 (a), node 1 (b), and node 2 (c)
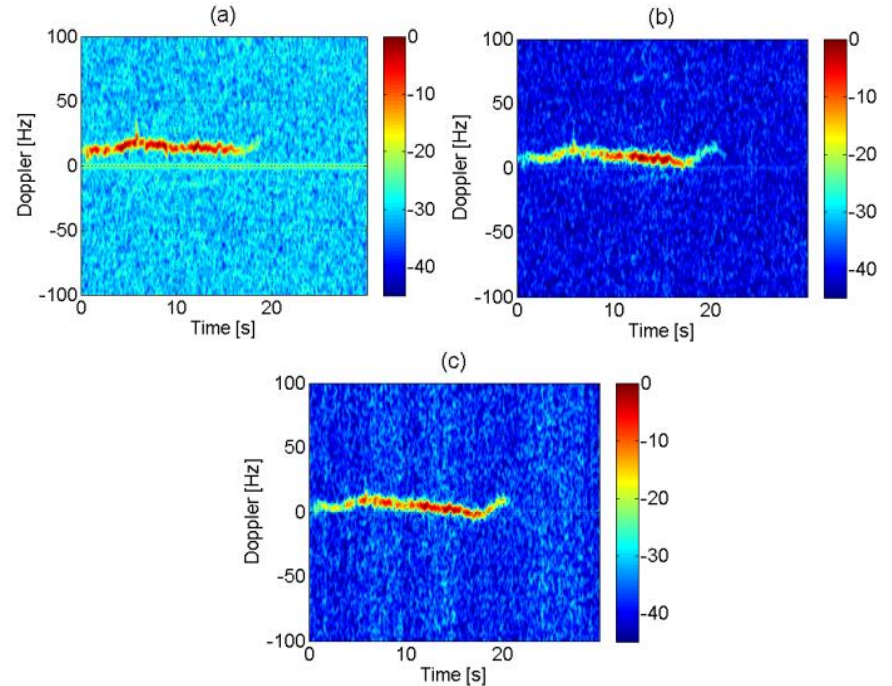

Figure 7 Time-Doppler plots of the 1st joint experiment as recorded at NetRAD node 3 (a), node 1 (b), and node 2 (c) 

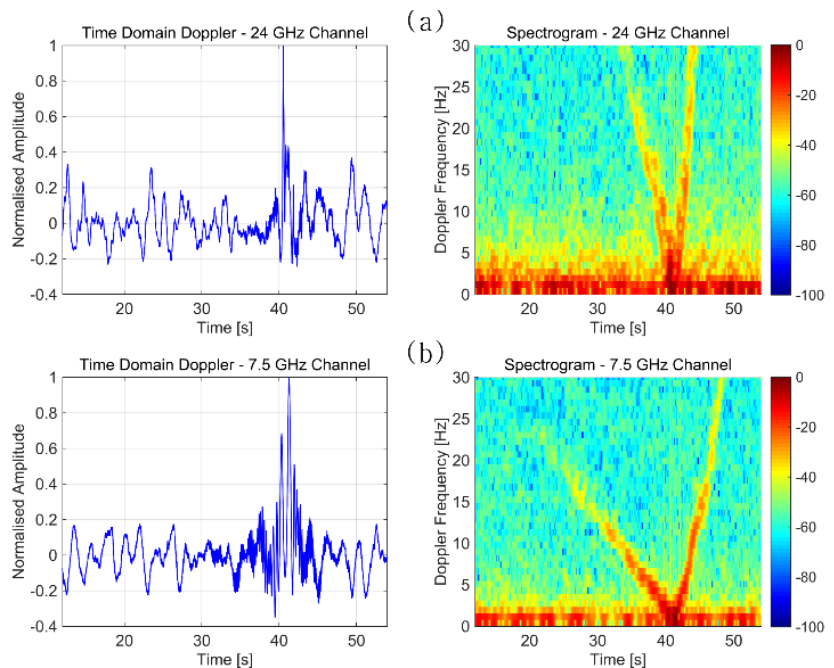

Figure 8 FSR target data for experiment 1. (a) and (b) show time domain Doppler and corresponding spectrograms for 24 and $7.5 \mathrm{GHz}$ radar channels respectively.

The FSR data in Fig. 8 shows for both radar frequencies in both the time and frequency domain the characteristic chirp of the target crossing the FSR baseline at a time 0.68 mins. From the spectrograms, the sea clutter can be seen to reside in the lower frequencies, less than $5 \mathrm{~Hz}$ [8], well separated from the higher target frequency components. The most obvious effects of the difference in carrier between (a) and (b) are the differing chirp rates in the spectrograms and the total target visibility time. The chirp rate ratio corresponds to the carrier frequency ratio. The visibility time difference is due to the effect of radar frequency on the FSCS main lobe, the higher frequency giving the narrower lobe [6-7] and thus lower visibility time at the receiver for a given target speed. It can be seen that for the $7.5 \mathrm{GHz}$ carrier, the visibility time is of the order of $30 \mathrm{~s}$. It is also noticeable that there is a difference in the chirp rate of the target signature either side of the zero Doppler baseline crossing point $41 \mathrm{~s}$ into the data record. This can be related as mentioned before, to the GPS data showing a variation in target speed either side of the baseline crossing. The motion parameter (trajectory and speed) extraction from the FSR signature is outside of the scope of this paper, a full description of this approach can be found in [6].

\subsection{Experiment 2 - Baseline Crossing Close to FSR Receiver (West Bank)}

In the second set of data presented here, the boat target again travels in a Northerly direction, this time however its trajectory is much closer to the West bank of the channel, where the multistatic equipment was deployed as well as the FSR receiver. 


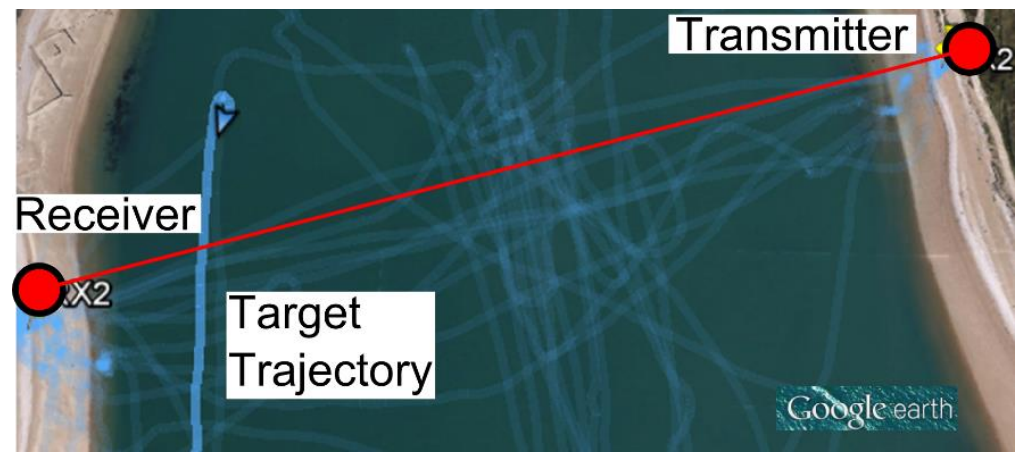

Figure 9 GPS track for second described trial of target crossing nearer to west bank of Langstone Harbour entrance.

Fig. 10 shows RTI plots of the target measurement for the multistatic system. Fig. 11 shows the corresponding micro-Doppler signatures and Fig. 12 the FSR signatures. As expected, in Fig. 10 the target appears much closer to the radar, at approximately 120-150 m 2-way range, as opposed to Fig. 6 where it was moving in the middle of the channel. The target appears much brighter in the monostatic plot (Fig. 10a), and rather weak in the bistatic plot of node 2 but still detectable (Fig. 10c). From Fig 11c the boat appears to be moving away from Node 2 with a speed of approximately $1.25 \mathrm{~m} / \mathrm{s}$ (corresponding to $-20 \mathrm{~Hz}$ Doppler), whereas in Fig. $11 \mathrm{~b}$ we can see the change of the main Doppler component from positive to negative as the boat approaches and moves away from node 1. With regards to the FSR data, in this case, in the time domain data it is hard to visualise the target signatures for both radar frequencies as they appear to be buried in the clutter (it is still required that we look at the test data more deeply to understand why there should be a dramatic increase in the clutter level/drop in the target signal for this particular measurement), however in the spectrogram the target is easily detectable in both cases.
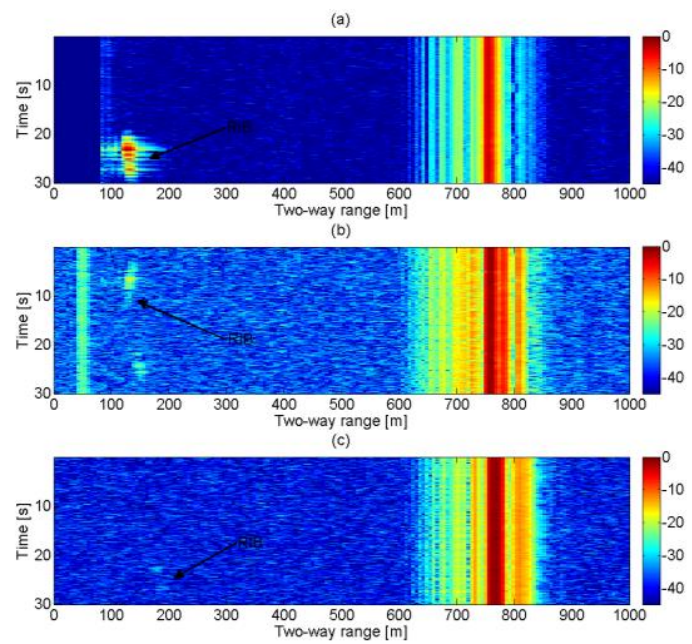

Figure 10 Range-Time plots of the second joint experiment as recorded at NetRAD node 3 (a), node 1 (b), and node 2 (c) 

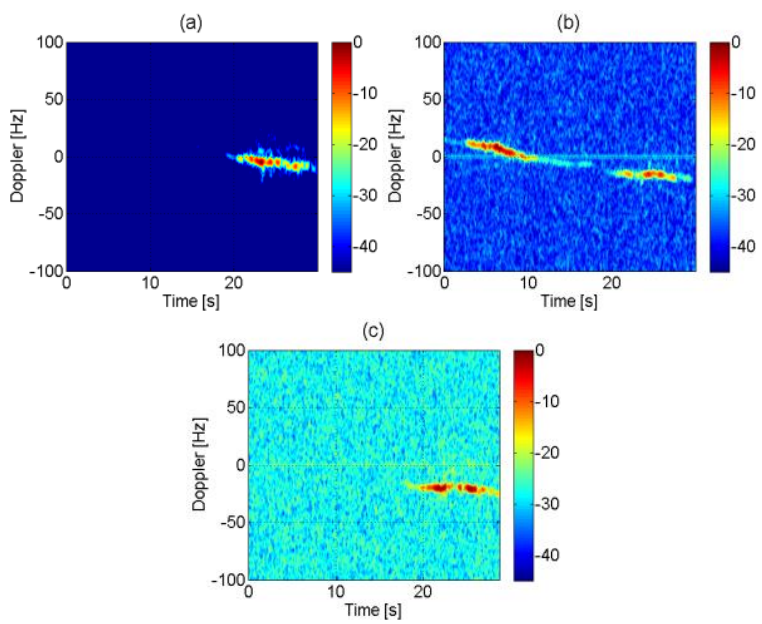

Figure 11 Time-Doppler plots of the second joint experiment as recorded at NetRAD node 3 (a), node 1 (b), and node 2 (c)
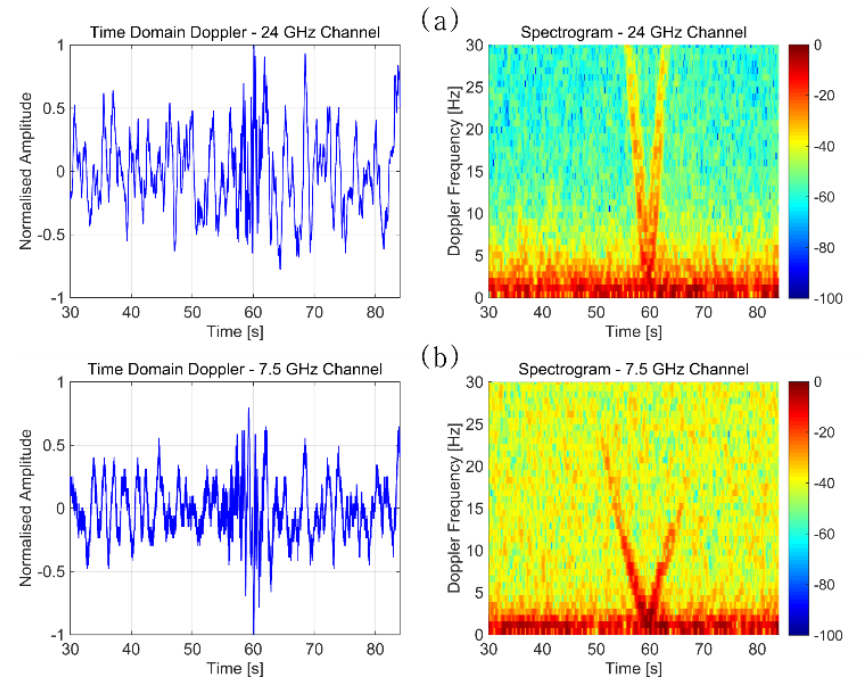

Figure 12 FSR experimental data for experiment 2. (a) and (b) show time domain Doppler and corresponding spectrograms for 24 and $7.5 \mathrm{GHz}$ radar channels respectively.

\subsection{Experiment 3 - Target moving away from monostatic node, towing inflatable ball}

In this third set of data, the RIB was moving away from the monostatic node and crossing the FS baseline from West to East with an angle of approximately $45^{\circ}$. The inflatable boat was also towing an additional target approximately $7.5 \mathrm{~m}$ behind, namely a plastic gym ball (diameter around $90 \mathrm{~cm}$ ). The target trajectory for this trial is shown in Fig. 13.

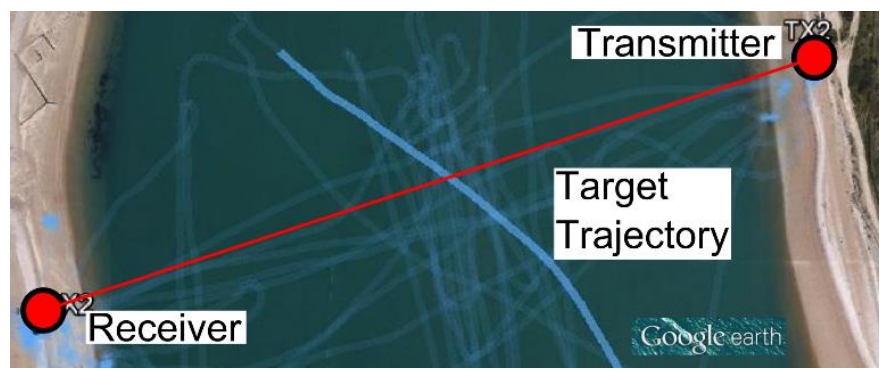

Figure 13 Target GPS tracks of target trajectory for third joint data set. 
In Fig. 14 the RTI plots of the third joint experiment are presented. The boat can be seen between $300 \mathrm{~m}$ and $400 \mathrm{~m}$ two-way range for all the three nodes. The ball appears to not be distinguishable from the boat. Fig. 15 shows the corresponding micro-Doppler signatures. As expected, the Doppler shifts are negative (inflatable moving away from the radar nodes) and their value is higher than in the previous micro-Doppler figures in Fig. 7 and 11, around $-50 \mathrm{~Hz}$ which corresponds to a speed of $3.13 \mathrm{~ms}^{-1}$. This is caused by the boat moving downstream, and also because the trajectory of the motion is such that all the velocity contributes to the Doppler measured at the monostatic node (the boat is moving away from this node along its line-of-sight). The Doppler shift is slightly lower at the multistatic node 1 and even more at node 2 , as expected from this geometry. From Fig. 16a, the $24 \mathrm{GHz}$ FSR channel, it is clear from both the time domain and spectrogram that two targets exist, and from the spectrogram these can be seen to cross the FSR baseline at times of 0.4 and 0.43 mins. Looking at Fig. 16b, the ball target is not visible, in either time domain or frequency domain for the $7.5 \mathrm{GHz}$ radar channel, this can possibly be described by the decreased FS cross section of the ball at the lower frequency. The similar shape of the two target chirps in the $24 \mathrm{GHz}$ spectrogram imply, as expected, that the trajectory of both targets is similar.

Using the speed for the target given by the GPS of approximately $4 \mathrm{~ms}^{-1}$ and the distance behind the boat of the ball being approximately $7.5 \mathrm{~m}$, this corresponds to the expected time difference between the ball and boat as shown in the spectrogram.

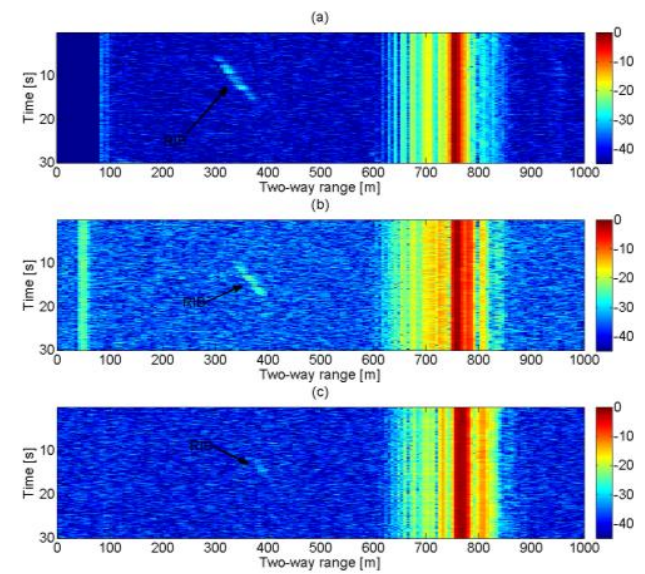

Figure 14 Range-Time plots of the third joint experiment as recorded at NetRAD node 3 (a), node 1 (b), and node 2 (c) 

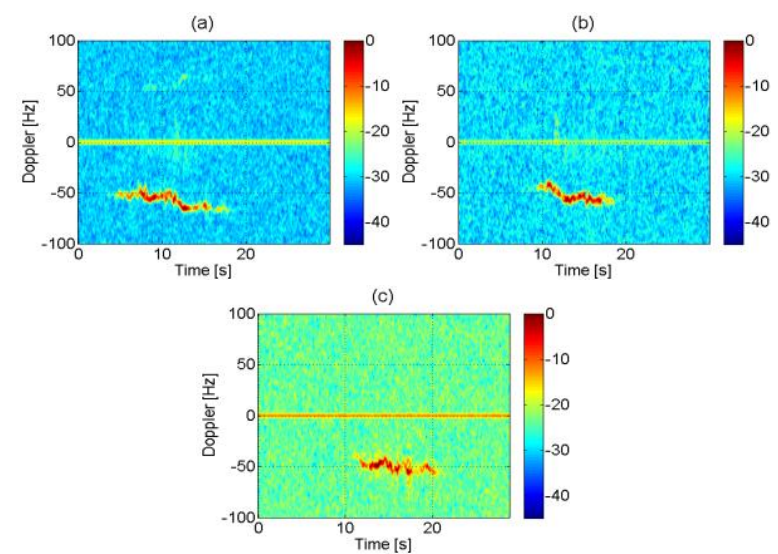

Figure 15 Time-Doppler plots of the third joint experiment as recorded at NetRAD node 3 (a), node 1 (b), and node 2 (c)
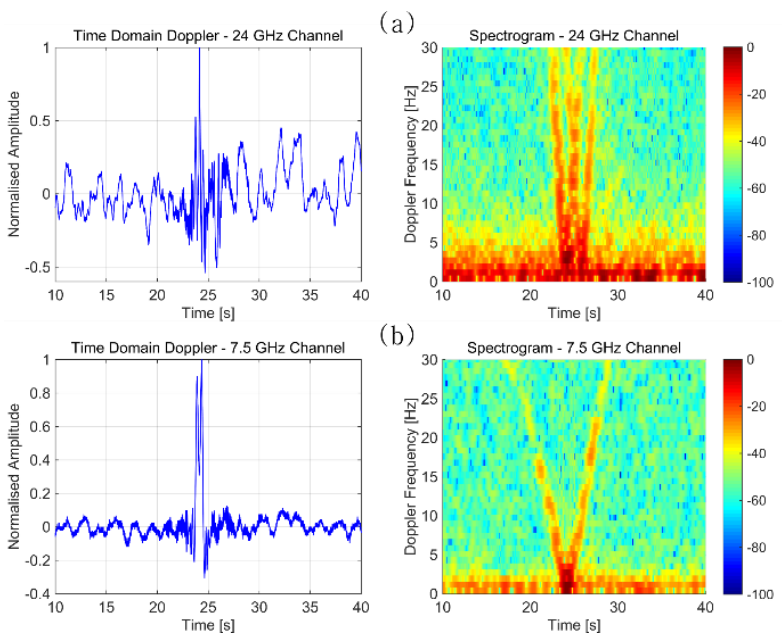

Figure 16 FSR experimental data for third experiment described. (a) and (b) show time domain Doppler and corresponding spectrograms for 24 and $7.5 \mathrm{GHz}$ radar channels respectively

\subsection{Comparison of with and without towed sphere target}

The range domain profiles from the three experiments both with and without a towed sphere target behind the small RIB boat have been further analysed. The datasets selected are named Joint Trial 1, 2, 3, 4, $5 \&$ 7. The towed sphere was behind the small rib at a distance of approximately $5 \mathrm{~m}$ for datasets 4,5 and 7 and there was no sphere towed behind the other three datasets. The average range profiles over the period the target was detectable (typically over a period of around $10 \mathrm{sec}$ ) from Node 3 (Monostatic node) can be seen in Figure 17a and 17b, for when a target was not towed or towed respectively. These profiles were evaluated by taking the average power of both clutter and target over the time period the target was present and then normalising the average clutter floor to be equal to $0 \mathrm{~dB}$, The two plots show comparable average power profiles in terms of the peak in the powers, with the towed target joint measurement 5 having the greatest peak. The shape of the averaged range profiles is quite different between these two situations, the non-towed target profile appears to have a smooth variation and general Gaussian shape, whereas the towed target profiles show much more fluctuations in the shorter range components of the profile. It is these small fluctuations that are likely to be induced by the towed sphere behind the inflatable 
boat. When observing the average SNR or the RTI plots themselves differentiating between the two scenarios is very challenging, but through the use of the averaged range profile it appears that this classification may be easier.

The Signal-to-Clutter (SCR) ratio of the six different experiments are also shown from all three radar nodes in Figure 18. This SCR was evaluated using a clutter limited patch of data and taking the mean peak signal power from the three adjacent range bins the target was present within. The values presented are not calibrated as a calibration setup was not used during the experiments undertaken. It is very interesting to note that for many of the examples shown the situation with no sphere target being towed produced higher SCR values in the two bistatic nodes (Nodes 1 and 2), whereas for the monostatic Node 3 , this was only found to be true on one occasion.

(a)

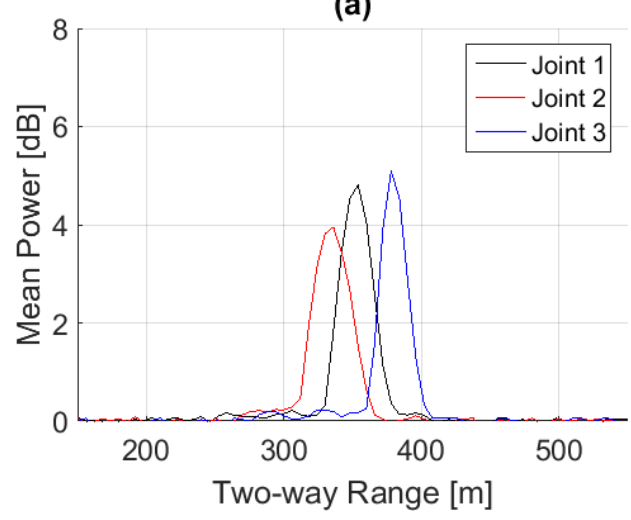

(b)

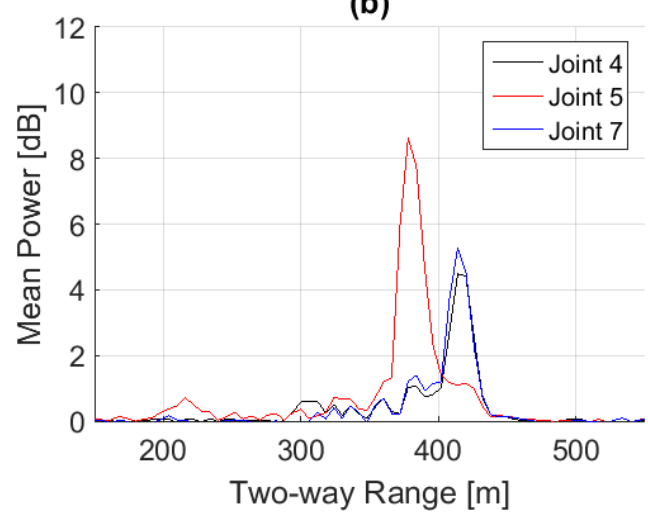

Figure 17 Average range profiles over time period boat target passes in front of Node 3 for (A) No towed sphere target (B) Towed sphere target

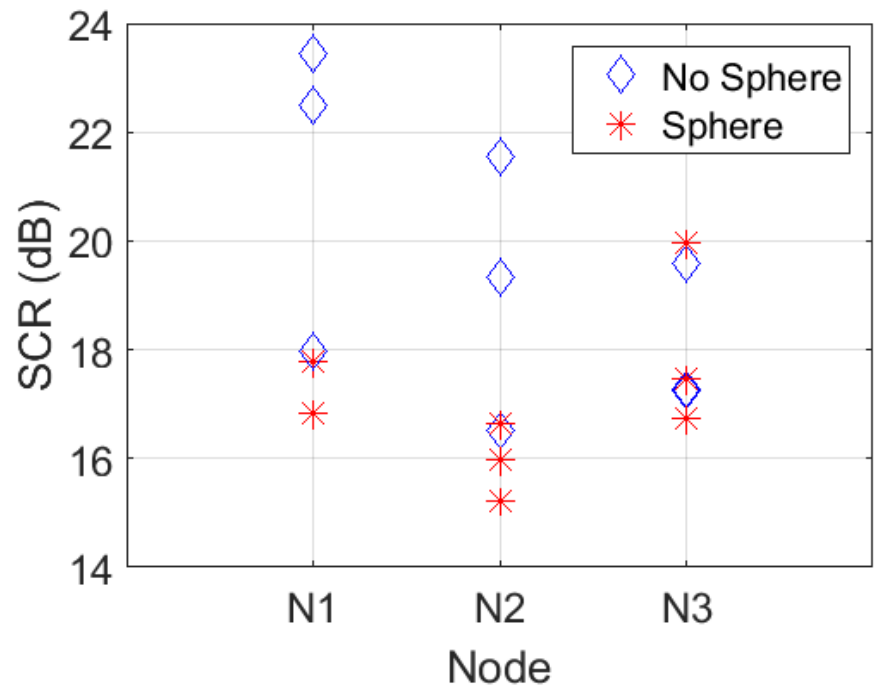

Figure 18. SCR levels for all three NetRAD Radar Nodes when measuring the target with and without the towed sphere As mentioned in Section 3.1, the signals from the FSR system are time domain Doppler signatures, which are formed in the regions close to and as targets cross the baseline. The formation is fundamentally due to 
the sweeping of the target forward scatter lobe pattern across the receive antenna [6]. In essence the target acts like a reradiating antenna creating a highly directive FSCS pattern dictated by the silhouette shape of the target itself, where the main lobe width $\theta_{\mathrm{fs}}^{\mathrm{ml}}$ and maximal FSCS are given by,

$$
\theta_{\mathrm{fs}}^{\mathrm{ml}} \approx \frac{\lambda}{D} \quad ; \quad \sigma_{\mathrm{fs}}^{\max }=4 \pi \frac{A^{2}}{\lambda^{2}}
$$

Where $\lambda$ is the radar wavelength, $D$ is the target's characteristic dimension and $A$ is the silhouette area of the target within the illuminating beam.

Due to this nature of the signal formation, any difference in time between baseline crossings of the targets i.e. boat towing a ball, will result in multiple temporally 'separate' signatures - this was seen in the spectrogram of the $24 \mathrm{GHz}$ data in Figure 16(a) and can be seen in the zoomed time domain signature (from record joint_7) in Figure 19. The FSR system is therefore able to separate these two targets in convoy.

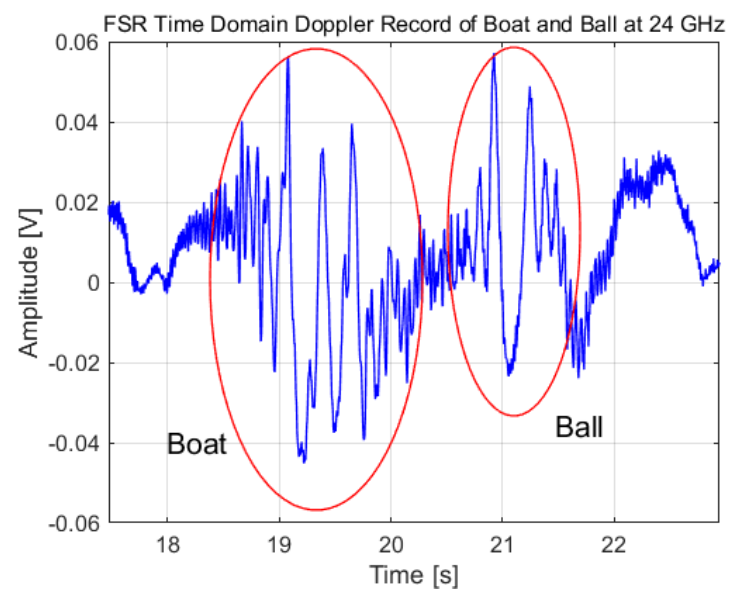

Figure 19 Zoomed FSR time domain Doppler signature of boat towing the ball. Targets are separated due to the difference in the FSR baseline crossing times.

Thus it is possible to look at the SCR's of the target and ball individually. In the raw Doppler data shown previously, it is clear that in the $7.5 \mathrm{GHz}$ channel the towed ball target was marginally visible (if at all) in the spectrogram data and so SCR calculations for the ball target are limited to the returns of the $24 \mathrm{GHz}$ channel.

The clutter power is estimated from the variance of a representative clutter section selected from the time domain Doppler signatures. Target (plus clutter) power is estimated through the $-3 \mathrm{~dB}$ point of the maximum of the squared time domain signals. Figure 20 shows an example $24 \mathrm{GHz}$ FSR Doppler signature with the selected sections of clutter, boat and ball targets. 


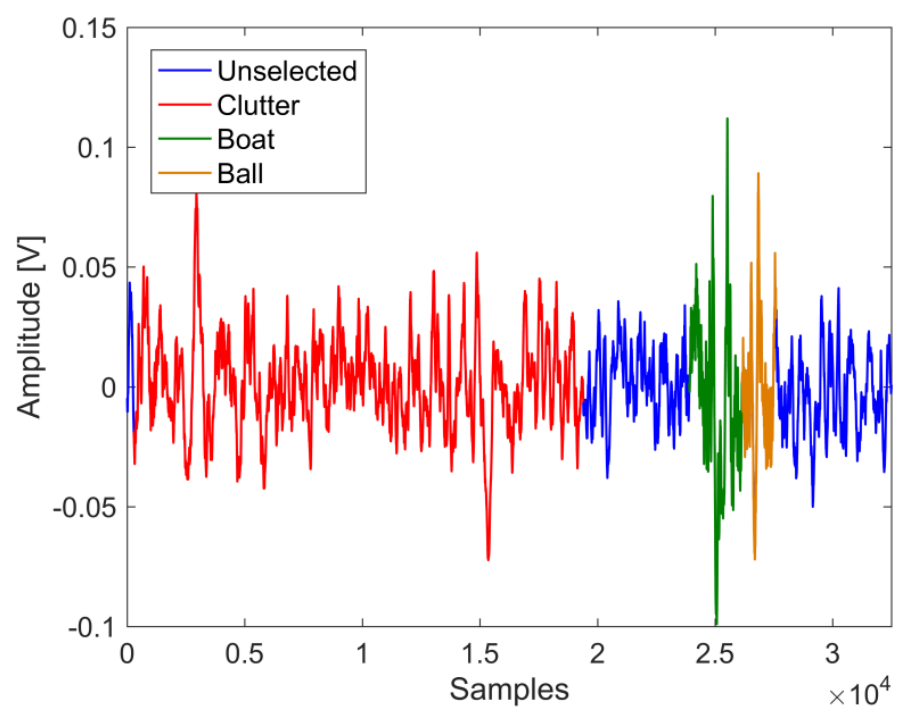

Figure 20 FSR time domain Doppler signature highlighting selected parts of signal for analysis: clutter, boat and ball targets.

It should be noted that in with this methodology, the SCR's are a first/worst case approximation. Figure 21 shows the comparison of the raw data signal to clutter ratios for the boat and the ball for the three measurements which contained both targets at $24 \mathrm{GHz}$. Similar marker style indicates the ball and boat are from the same measurement.

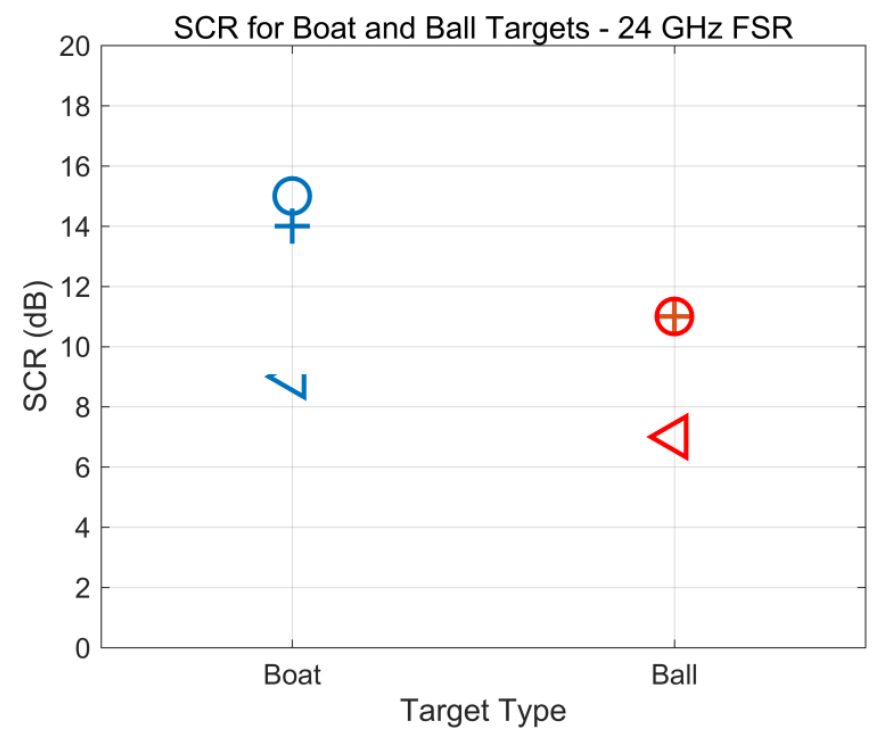

Figure 21 Comparison of SCR's for the FSR $24 \mathrm{GHz}$ measurements of boat and ball target. Similar markers indicate measurement from a single data record of the boat towing the ball.

As would be expected, within a given record, the ball has a reduced signal to clutter ratio in comparison to the boat. This is due to the lower FSCS of the ball target due to its smaller silhouette area in relation to the boat, as described in equation (1) - a comparable reduction is observed between the three measurements. There is one record that for both targets shows a much reduced SCR in comparison to the other 
measurements. A general rise in clutter level may be responsible for this, it should be noted however that general variation in SCR, especially when assessed by the method above, should be apparent. FSR operates at very low grazing angles for surface target detection, in order to take advantage of the increased FSCS from a directive narrow FS lobe. Variations in target height caused by the underlying sea waves can impact the received signal strength from the target.

One simplistic method of improving SCR in maritime FSR is through knowledge of the clutter spectrum. In [11] it was shown that in maritime FSR, the clutter spectral width is generally invariant to experimental and environmental parameters, being restricted to below $1 \mathrm{~Hz}$. This allows the design of a high pass clutter/whitening filter. Obviously the advantages of using this method are very dependent on the speed and trajectory of the target. Ultimately, coherent (matched filter) processing should be applied and for FSR this procedure was outlined in [6], through correlation with a set of reference waveforms - the application in this case was for estimation of target motion parameters

\section{Conclusions}

In this paper we described and discussed a set of joint trials completed in an attempt to provide a simultaneously measured data set from both multistatic and forward scatter radars to allow the comparison for the detection of small/difficult maritime targets such as inflatable rubber boats. Both radar systems were shown to be capable of detecting a small boat target, using very different approach to achieve this.

A qualitative/descriptive analysis of the target signatures using both systems has been presented with reference to a few examples of the joint measurements where the boat was performing different movements. Range-Time plots and micro-Doppler signatures at the three radar nodes have been shown for the multistatic data, and time domain Doppler and spectrogram plots for the FSR at two frequencies.

These preliminary results and process of joint experimentation have highlighted the next stages required and some issues that need to be addressed in further work to establish a more quantitative comparison of the two systems and investigate detection advantages over conventional monostatic radar. For instance the alignment of the antenna beams of the multistatic radar needs to be improved to avoid the target being outside the coverage area and therefore not detectable. In addition, an improved method of synchronizing the recording from the multistatic radar and the motion of the target needs to be developed exploiting the fact that the inflatable boat is GPS-instrumented. This would allow us to investigate multistatic tracking of the target and compare back to the ground truth provided by the GPS signal.

Additional analysis has focused on the differences observed between a small inflatable boat with and without a towed spherical target behind it. This target was very small and the dominant changes in the 
responses observed are potentially due to contributions from the wake of the overall boat and sphere pair. The monostatic average range profiles as the boat was observed have shown subtle differences in their structure that may allow for discrimination between these two situations. The actual SCR values obtain were found to be very similar with and without the towed target behind the boat, and in fact a great SCR value was found in the bistatic case when not towing the target. With regards to the FSR signals, due to the nature of the target signature formation, the target and ball can be resolved separately in time in the 24 GHz records and so SCR analysis may be performed on the targets individually. The measurements show an expected decrease in the SCR for the ball compared to the boat and overall good SCR's are realised even though the calculation has been performed on the raw Doppler data before any form of non-coherent or coherent processing.

There is always the added complication of comparing such systems in respect that in order to obtain cross section enhancement in FSR it is necessary to work in the upper Mie and optical scattering regimes. Therefore both of the systems we are comparing are functioning in different bands and comparisons must be made with this fact in mind.

This experiment represents the first set of recorded data using both radar types, this data can now be used to begin to make estimations of system performance and also allows us to make decisions on improvements required for future trials.

\section{Acknowledgement}

This work was funded by the EPSRC grant EP/J008419/1. The authors would like to thank Dr. Amin Amiri and Mr. Saad Alhuwaimel for their precious help during the experimental trials.

\section{References}

[1] Ward, K.; Tough, R.; Watts, S.; "Sea Clutter - Scattering, the K Distribution and Radar Performance, 2nd edition", the Institution of Engineering and Technology, 2013.

[2] International Maritime Organization Resolution MSC.192(79), “Adoption of the Revised Performance Standards for Radar Equipment”, December 2004.

[3] Al-Ashwal, W.A.; Griffiths, H.D., "Preliminary analysis of monostatic and bistatic Doppler signature of small maritime target," International Radar Conference, Adelaide, Australia, 9-12 Sept. 2013.

[4] Inggs, M.; Griffiths, H.D.; Fioranelli, F.; Ritchie, M.; Woodbridge, K., "Multistatic radar: System requirements and experimental validation," International Radar Conference, Lille, France, 13-17 Oct. 2014. 
[5] Daniel, L.Y.; Hoare, E.G.; Gashinova, M.; Svintsov, A.; Cherniakov, M.; Sizov, V., "Ultrawideband forward scatter radar fence for maritime surveillance — Initial experimental results," IEEE Radar Conference, pp.526-531, Washington DC, USA. 10-14 May 2010.

[6] Gashinova, M.; Daniel, L.; Sizov, V.; Hoare, E.; Cherniakov, M., "Phenomenology of Doppler forward scatter radar for surface targets observation," IET Radar, Sonar \& Navigation, vol.7, no.4, pp.422,432, April 2013.

[7] Gashinova, M.; Daniel, L.; Hoare, E.; Sizov, V.; Kabakchiev, K.; Cherniakov, M., "Signal characterisation and processing in the forward scatter mode of bistatic passive coherent location systems," EURASIP Journal on Advances in Signal Processing 2013, 2013:36.

[8] Kabakchiev, K.; Daniel, L.; Gashinova, M.; Hoare, E.; Cherniakov, M.; Sizov, V., "Radar parameters influence on the clutter in maritime forward scatter radar", 2014 11th European Radar Conference (EuRAD), pp.113-116, Rome, Italy, 8-10 Oct. 2014.

[9] Al-Ashwal, W.A.; Baker, C.J.; Balleri, A.; Griffiths, H.D.; Harmanny, R.; Inggs, M.; Miceli, W.J.; Ritchie, M.; Sandenbergh, J.S.; Stove, A.; Tough, R.J.A.; Ward, K.D.; Watts, S.; Woodbridge, K., "Statistical analysis of simultaneous monostatic and bistatic sea clutter at low grazing angles", Electronics Letters, vol.47, no.10, pp.621,622, May 122011.

[10] Fioranelli, Francesco; Ritchie, Matthew; Griffiths, Hugh: 'Multistatic human micro-Doppler classification of armed/unarmed personnel', IET Radar, Sonar \& Navigation, vol. 9, no. 7, p. 857-865, August 2015.

[11] Gashinova, M.; Kabakchiev, K.; Daniel, L.; Hoare, E.; Sizov, V.; Cherniakov, M., "Measured forward-scatter sea clutter at near-zero grazing angle: analysis of spectral and statistical properties," IET Radar, Sonar \& Navigation, vol. 8, no. 2, pp. 132-141, February 2014. 